\title{
VARIABILIDADE GENÉTICA E SELEÇÃO PARA CARACTERES DE CRESCIMENTO DA SERINGUEIRA $\left({ }^{1}\right)$
}

\author{
REGINALDO BRITO DA COSTA $\left({ }^{2 *}\right)$; MARCOS DEON VILELA DE RESENDE $\left({ }^{3}\right)$; \\ PAULO DE SOUZA GONÇALVES $\left({ }^{4}\right)$; JOSÉ FRANKLIM CHICHORRO $\left({ }^{5}\right)$; \\ RAUL ALFFONSO RODRIGUES ROA $\left({ }^{6}\right)$
}

\begin{abstract}
RESUMO
O presente estudo objetivou estimar parâmetros e valores genéticos para os caracteres altura e diâmetro de progênies de seringueira, pelo método de modelos mistos (procedimento REML/BLUP). Progênies de meio-irmãos foram estabelecidas em área do município de Dois Irmãos do Buriti (MS), sob delineamento de blocos ao acaso, com 28 tratamentos (progênies), cinco repetições e dez plantas por parcela. A coleta dos dados foi realizada em março de 2004. Aos 12 meses de idade foram avaliados os caracteres altura e diâmetro. Nas herdabilidades individuais no sentido restrito para altura $(0,1877)$ e diâmetro $(0,1809)$, bem como nas herdabilidades médias no sentido restrito de progênies para altura $(0,3385)$ e diâmetro $(0,4124)$ observaram-se boas magnitudes. Ganhos genéticos preditos na ordem de $14,04 \%$ a $16,54 \%$ foram obtidos para o caráter altura e $25,76 \%$ a 91,78\% para o caráter diâmetro. Os resultados estimulam a continuidade do programa de melhoramento genético, com a possibilidade de maximização dos ganhos nas gerações seguintes.
\end{abstract}

Palavras-chave: Hevea brasiliensis, parâmetros genéticos, ganho genético, procedimento REML/BLUP.

\section{ABSTRACT \\ GENETIC VARIABILITY AND SELECTION FOR GROWTH TRAITS OF RUBBER TREE}

This study aim to estimate genetic values and parameters for height and diameter of rubber tree plants by the methodology of mixed models (REML/BLUP procedure). Half-sib progenies were established set Dois Irmãos do Buriti, Mato Grosso do Sul State in complete randomised block, with 28 treatments, 5 replications and 10 plants per plot. On March, 2004, twelve months after transplanting, plant height and diameter were evaluated. The individual narrow sense heritability for plant height (0.1877) and diameter (0.4124) showed good magnitude. Estimated genetic gains of from $14.04 \%$ to $16.54 \%$ were obtained for height and $25.76 \%$ to $91.78 \%$ for diameter indicating the possibility of selection for those traits.

Key words: Hevea brasiliensis, genetic parameters, genetic gain, REML/BLUP procedure.

$\left({ }^{1}\right)$ Trabalho realizado com apoio financeiro do CNPq e CAPES. Recebido para publicação em 17 de abril e aceito em 18 de outubro de 2007.

$\left({ }^{2}\right)$ Universidade Católica Dom Bosco (UCDB), Programa de Mestrado em Desenvolvimento Local, Av. Tamandaré, 6000, Jardim Seminário, Caixa Postal 100, 79117-100 Campo Grande (MS). E-mail: rcosta@ucdb.br ( ${ }^{*}$ ) autor correspondente.

$\left({ }^{3}\right)$ Embrapa-Centro Nacional de Pesquisa de Florestas (CNPF), Caixa Postal 31, 83411-000 Colombo (PR). E-mail: deon@cnpf.embrapa.br

$\left({ }^{4}\right)$ Embrapa-Programa Seringueira, Instituto Agronômico (IAC), Caixa Postal 28, 13012-970 Campinas (SP). E-mail: paulog@iac.sp.gov.br

$\left({ }^{5}\right)$ Programa de Mestrado em Ciências Florestais e Ambientais, Faculdade de Engenharia Florestal da Universidade Federal de Mato Grosso (UFMT). Av. Fernando Corrêa da Costa, s/n, Coxipó, 78060-900 Cuiabá (MT). E-mail: jfranklim@terra.com.br

( $\left.{ }^{6}\right)$ Mestrando do Programa Desenvolvimento Local, Universidade Católica Dom Bosco (UCDB), Campo Grande (MS). Bolsista CAPES. 


\section{INTRODUÇÃO}

A seringueira [Hevea brasiliensis (Willd ex Adr. de Juss.) Muell.-Arg.], tem como principais áreas de cultivo, no Brasil, as denominadas áreas de escape do mal-das-folhas e localizam-se nos seguintes Estados: São Paulo, Mato Grosso, Mato Grosso do Sul, Bahia e no noroeste do Paraná (Pereira, 1992).

Diversos trabalhos que visam maximizar os ganhos genéticos da seringueira têm sido realizados no Sri Lanka (JAYASEKERA, 1983), Indonésia (DASLIN et al., 1986), Nigéria (ONOKPISE et al., 1986), Índia (Meenattor et al., 1991), Malásia (TAN, 1995) e Brasil (GONÇALVES et al., 1998; COSTA et al., 2000 a; GONÇALVES et al., 2005; FURLANI et al., 2005), dentre outros.

Os testes de progênies, instrumentos importantes para o trabalho do melhorista, têm sido usados na estimação de parâmetros genéticos e seleção de indivíduos, quando se procura avaliar a magnitude e a natureza da variância genética disponível com vistas a quantificar e maximizar os ganhos genéticos, utilizando-se procedimento de seleção adequado.

A acurada predição de valores individuais de candidatos para seleção é parte essencial em programas de melhoramento genético florestal (RESENDE, 2002a). O método Reml/Blup, desenvolvido para o melhoramento de plantas perenes tem maximizado os ganhos genéticos com seleção (RESENDE, 2002b, Costa et al., 2002; Costa et al., 2005; Missio et al., 2005), por se tratar de um procedimento estimativo, especialmente para dados desbalanceados, predizendo valores genéticos dos indivíduos em testes de progênies (RESENDE e FERnANDES, 1999).

Este estudo objetivou estimar parâmetros e valores genéticos para os caracteres altura e diâmetro de progênies de seringueira pela método de modelos mistos (procedimento REML/BLUP). Tais estimativas visam subsidiar o primeiro programa de melhoramento genético da espécie no Estado de Mato Grosso do Sul.

\section{MATERIAL E MÉTODOS}

O ensaio foi instalado em área pertencente à prefeitura do município de Dois Irmãos do Buriti (MS), localizada a $20^{\circ} 29^{\prime} 14^{\prime \prime} \mathrm{S}$ e $55^{\circ} 30^{\prime} 40^{\prime \prime} \mathrm{W}$ e altitude de $320 \mathrm{~m}$. A temperatura média anual é de $27^{\circ} \mathrm{C}$ e a pluviosidade média anual é de $1.370 \mathrm{~mm}$.

O material genético que compõe o estudo é constituído de 28 progênies de meios-irmãos provenientes de uma população de segunda geração produzida de recombinação de 28 matrizes. A população de recombinação está estabelecida no Pólo Noroeste Paulista, Votuporanga (SP), pertencente à APTA/SAA.

O experimento foi estabelecido sob delineamento de blocos ao acaso com 28 tratamentos (progênies) três repetições e dez plantas por parcela em linhas simples, no espaçamento $3 \times 2 \mathrm{~m}$.

Aos 12 meses de idade, as progênies foram avaliadas quanto aos caracteres: a) altura total das plantas $(\mathrm{cm})$ e b) diâmetro na base do caule $(\mathrm{mm})$.

As variáveis foram analisadas usando-se o método de modelo linear misto univariado aditivo (modelo 1) do software SELEGEN - REML/BLUP apresentado por RESENDE (2002b), consistindo do seguinte:

$$
\mathrm{y}=\mathrm{Xb}+\mathrm{Za}+\mathrm{Wc}+\mathrm{e}, \text { em que: }
$$

$y, b, a, c$ e e: vetores de dados, dos efeitos das médias de blocos (aleatório), de efeitos genéticos aditivos (aleatório), de efeitos de parcela (aleatório) e de erros aleatórios, respectivamente.

$X, Z$ e W: matrizes de incidência para $b$, a e c respectivamente.

Distribuições e estruturas de médias e variâncias

$$
\begin{aligned}
& y \mid b, V \sim N(X b, V) \\
& a \mid A, \hat{\sigma}_{A}^{2} \sim N\left(0, A \sigma_{a}^{2}\right) \\
& c \mid \hat{\sigma}_{c}^{2} \sim N\left(0, I \sigma_{c}^{2}\right) \\
& e \mid \hat{\sigma}_{e}^{2} \sim N\left(0, I \sigma_{e}^{2}\right) \\
& \operatorname{Cov}\left(a, c^{\prime}\right)=0 ; \quad \operatorname{Cov}\left(a, e^{\prime}\right)=0 ; \quad \operatorname{Cov}\left(c, e^{\prime}\right)=0
\end{aligned}
$$

ou seja:

$$
\begin{aligned}
& \mathrm{E}\left[\begin{array}{l}
\mathrm{y} \\
\mathrm{a} \\
\mathrm{c} \\
\mathrm{e}
\end{array}\right]=\left[\begin{array}{c}
\mathrm{Xb} \\
0 \\
0 \\
0
\end{array}\right] \text { e } \operatorname{Var}\left[\begin{array}{l}
\mathrm{y} \\
\mathrm{a} \\
\mathrm{c} \\
\mathrm{e}
\end{array}\right]=\left[\begin{array}{cccc}
\mathrm{V} & \mathrm{ZG} & \mathrm{WC} & \mathrm{R} \\
\mathrm{G} Z & \mathrm{G} & 0 & 0 \\
\mathrm{CW} & 0 & \mathrm{C} & 0 \\
\mathrm{R} & 0 & 0 & \mathrm{R}
\end{array}\right] \text {, em que: } \\
& G=A \quad \hat{\sigma}_{A}^{2} \\
& R=I \hat{\sigma}_{c}^{2} \\
& C=I \hat{\sigma}_{e}^{2} \\
& V=Z A \hat{\sigma}_{a}^{2} Z^{\prime}+W I \hat{\sigma}_{c}^{2} W^{\prime}+I \hat{\sigma}_{e}^{2}=Z G Z^{\prime}+W C W^{\prime}+R .
\end{aligned}
$$

Equações de modelo misto

$$
\begin{aligned}
& {\left[\begin{array}{ccc}
X^{\prime} X & X^{\prime} Z & X^{\prime} \mathrm{W} \\
Z^{\prime} X & Z^{\prime} Z+A^{-1} \lambda_{1} & Z^{\prime} \mathrm{W} \\
\mathrm{W}^{\prime} \mathrm{X} & \mathrm{W}^{\prime} \mathrm{Z} & \mathrm{W}^{\prime} \mathrm{W}+\mathrm{I} \lambda_{2}
\end{array}\right]\left[\begin{array}{l}
\hat{\mathrm{b}} \\
\hat{\mathrm{a}} \\
\hat{\mathrm{c}}
\end{array}\right]=\left[\begin{array}{c}
\mathrm{X}^{\prime} \mathrm{y} \\
\mathrm{Z}^{\prime} \mathrm{y} \\
\mathrm{W}^{\prime} \mathrm{y}
\end{array}\right] \text {, em que: }} \\
& \lambda_{1}=\frac{\hat{\sigma}_{e}^{2}}{\hat{\sigma}_{a}^{2}}=\frac{1-\hat{h}^{2}-c^{2}}{\hat{h}^{2}} ; \quad \lambda_{2}=\frac{\hat{\sigma}_{e}^{2}}{\hat{\sigma}_{a}^{2}}=\frac{1-\hat{h}^{2}-\hat{c}^{2}}{\hat{c}^{2}}
\end{aligned}
$$


$\hat{h}_{a}^{2}=\frac{\hat{\sigma}_{a}^{2}}{\hat{\sigma}_{a}^{2}+\hat{\sigma}_{c}^{2}+\hat{\sigma}_{e}^{2}}=$ herdabilidade individual no sentido

restrito no bloco;

$\hat{h}_{m p}{ }^{2}=\frac{0,25 \hat{\sigma}_{a}^{2}}{0,25 \hat{\sigma}_{a}^{2}+\hat{\sigma}_{c}^{2} / b+\hat{\sigma}_{e}^{2} /(n b)}=$ herdabilidade média de progênies no sentido restrito no bloco;

$\hat{c}^{2}=\hat{\sigma}_{c}^{2} /\left(\hat{\sigma}_{a}^{2}+\hat{\sigma}_{c}^{2}+\hat{\sigma}_{e}^{2}\right)=$ correlação devida ao

ambiente comum da parcela;

$\hat{\sigma}_{a}^{2}=$ variância genética aditiva;

$\hat{\sigma}_{\text {parc }}^{2}=$ variância entre parcelas;

$\mathrm{CV}_{\mathrm{g}}(\%)=\frac{\sqrt{\hat{\sigma}_{a}^{2}}}{\bar{X}} \cdot 100$

$\mathrm{CV}_{\mathrm{e}}(\%)=\frac{\sqrt{\hat{\sigma}_{e}^{2}}}{\bar{X}} \cdot 100$

$\hat{\sigma}_{e}^{2}=$ variância residual dentro da parcela (ambiental + não aditiva);

$\mathrm{A}=$ matriz de correlação genética aditiva entre os indivíduos em avaliação.

Acurácia seletiva $=$ obtida através da raiz quadrada da herdabilidade média de progênie.

As estimativas dos parâmetros genéticos foram obtidas pelo procedimento REML (restricted maximum likelihood) a partir de iterações nas equações de modelo misto.

\section{RESULTADOS E DISCUSSÃO}

Os resultados referentes às estimativas dos parâmetros genéticos para os caracteres altura e diâmetro das plantas são apresentados na tabela 1 .

Nos coeficientes de variação genética individual $\left(\mathrm{CV}_{\mathrm{g}} \%\right)$, que expressam em percentagem da média geral a quantidade de variação genética existente, observaramse valores moderados para os caracteres diâmetro e altura $(16,42 \%$ e $11,35 \%)$ respectivamente. Esses resultados são condizentes àqueles obtidos por MORETI et al. (1994), Boock et al. (1995) e CosTA et al. (2000a) e revelam que a população pode ser considerada apropriada para o programa de melhoramento genético. Em outros termos, ganho genético é esperado aplicandose procedimento adequado de seleção.

Para os coeficientes de variação residual dentro da parcela $\left(\mathrm{CV}_{\mathrm{e}} \%\right)$, os valores são de $13,74 \%$ para altura e $16,97 \%$ para diâmetro, considerados de baixa magnitude para os respectivos caracteres por GARCIA et al. (1989).
Tabela 1. Estimativas de parâmetros genéticos para os caracteres altura e diâmetro em progênies de seringueira, no município de Dois Irmãos do Buriti (MS)

\begin{tabular}{lcc}
\hline Estimativas $\left(^{1}\right)$ & Altura & Diâmetro \\
\hline$\hat{\mathrm{h}}_{\mathrm{a}}^{2}$ & $\mathrm{~cm}$ & $\mathrm{~mm}$ \\
$\hat{h}_{m p}^{2}$ & 0,1877 & 0,1809 \\
$\hat{\sigma}_{a}^{2}$ & 0,3385 & 0,4124 \\
$\hat{\sigma}_{p a r c}^{2}$ & 113,6164 & 4,8305 \\
$\hat{\sigma}_{e}^{2}$ & 107,8686 & 2,2576 \\
$\hat{\sigma}_{f}^{2}$ & 383,7686 & 19,6003 \\
\hline Média geral $^{2}$ & 605,2537 & 26,6885 \\
$(\mathrm{CV}$ g\%) & 93,8866 & 13,3795 \\
$(\mathrm{CV} \%)$ & 11,35 & 16,42 \\
\hline
\end{tabular}

$\left({ }^{1}\right)$ Herdabilidade individual no sentido restrito no bloco $\left(\hat{\mathrm{h}}_{\mathrm{a}}^{2}\right)$, herdabilidade média de progênie $\left(\hat{h}_{m p}^{2}\right)$, variância genética aditiva $\left(\hat{\sigma}_{a}^{2}\right)$, variância ambiental entre parcelas $\left(\hat{\sigma}_{\text {parr }}^{2}\right)$, variância residual dentro de parcela (ambiental + não aditiva, $\left.\hat{\sigma}_{e}^{2}\right)$, variância fenotípica individual $\left(\hat{\sigma}_{f}^{2}\right)$, coeficiente de variação genética $\left(\mathrm{CV}_{\mathrm{g}} \%\right)$, coeficiente de variação ambiental dentro de parcelas $\left(\mathrm{CV}_{\mathrm{e}} \%\right)$.

As estimativas dos coeficientes de herdabilidades individuais no sentido restrito $(0,1877$ e 0,1809 ) foram baixas, mas pode propiciar ganhos genéticos com seleção na seqüência das avaliações. Estes resultados são condizentes com aqueles observados por PAIVA et al. (1982), AlikA (1985) e Costa et al. (2000a) para a seringueira. Ressaltam-se os valores mais altos das herdabilidades de médias de progênies $(0,3385$ e 0,4124$)$ para os caracteres em estudo, revelando que a seleção pode ser mais efetiva utilizando-se as informações das famílias. Tais resultados são coerentes com os observados na literatura relativa à seringueira (GILBERT et al., 1973; Nga e Subramanian, 1974; Moreti et al., 1994, Costa et al., 2000b e GONÇALVES et al., 2005).

A mais importante função da herdabilidade no estudo genético do caráter métrico é seu papel preditivo expressando a confiança do valor fenotípico como um guia para o valor genético, ou o grau de correspondência entre o valor fenotípico e o valor genético (FALCONER, 1987; VENCOVSKY e BARRIGA, 1992). Segundo FAlCONER (1987), a herdabilidade é uma propriedade não somente de um caráter, mas também da população e das circunstâncias de ambientes às quais os indivíduos estão sujeitos. O valor da herdabilidade poderá ser afetado se houver alteração em qualquer um dos componentes das variâncias genética e fenotípica. 
Na tabela 2, são apresentados os valores fenotípicos, genéticos aditivos, ganhos genéticos preditos e nova média da população, das 10 melhores indivíduos para o caráter altura $(\mathrm{cm})$.

Constata-se que os indivíduos das três melhores progênies $(8,21$ e 2$)$, são promissores quando comparados aos indivíduos das demais progênies. Os ganhos genéticos variaram de $14,04 \%$ a $16,54 \%$, elevando a nova média da população, após um ciclo de seleção, de 93,88 cm para 109,42 $\mathrm{cm}$ do indivíduo 2 da progênie 8. Portanto, estimulam a utilização desses germoplasmas, na seqüência do programa de melhoramento genético, incluindo-se o caráter produção de borracha nas futuras avaliações.

A acurácia ou correlação entre os valores genéticos preditos e os verdadeiros foi de 0,58 para altura e 0,64 para diâmetro (Tabelas 2 e 3). Segundo Resende (2002a), a acurácia é uma medida que está associada à precisão na seleção, e é o principal componente do progresso genético que o melhorista pode alterar visando maximizar o ganho genético. A acurácia pode ser incrementada por meio de uma experimentação mais adequada, mantendo-se o mesmo tamanho do experimento, porém alterando-se o número de indivíduos por parcela e de repetições (RESENDE 2002a).

As acurácias encontradas neste trabalho são condizentes com aquelas apresentadas por Costa et al. (2000b) em seringueira (para produção de borracha).
Na Tabela 3, são relacionados os valores fenotípicos, genéticos aditivos ganhos preditos e nova média da população, dos 10 melhores indivíduos para o caráter diâmetro ( $\mathrm{mm}$ ) em seringueira, no Município de Dois Irmãos do Buriti (MS).

Observa-se que os indivíduos da progênie 20 foram os mais promissores, com valores expressivos e constituem os nove melhores materiais genéticos classificados, para o caráter diâmetro. De forma similar ao caráter altura, avaliações posteriores em idades mais avançadas poderão confirmar o desempenho desses materiais para efeito de seleção, visando maximizar o ganho genético. SIMEÃo et al. (2002) enfatizam que os valores genéticos preditos em relação a todos os indivíduos candidatos possibilitam estabelecer a melhor estratégia para o aumento da eficiência do melhoramento.

$\mathrm{Na}$ tabela 4 , verificam-se os valores genéticos aditivos preditos $(\hat{\mu}+\hat{a})$ e genotípicos $(\hat{\mu}+\hat{g})$, assumindo-se grau médio de dominância igual a $1 \mathrm{em}$ uma população com nível intermediário de melhoramento dos 10 melhores genótipos da seringueira aos 12 meses de idade para o caráter altura.

Verifica-se que dentre o material genético selecionado, são comuns os melhores indivíduos para propagação sexuada (ordenados por $\hat{\mu}+\hat{a}$ ) e assexuada (ordenados por $\hat{\mu}+\hat{g}$ ), porém a seqüência dos indivíduos é alterada pelo tipo de propagação considerada, o que também foi observado por RESENDE e Dias (2000) para o caráter número de frutos por planta, em progênies de cacau.

Tabela 2. Valores fenotípicos, genéticos aditivos, ganhos genéticos preditos e nova média da população melhorada, das 10 melhores progênies para o caráter altura $(\mathrm{cm})$ de seringueira, no Município de Dois Irmãos do Buriti (MS)

\begin{tabular}{|c|c|c|c|c|c|c|}
\hline Bloco & Progênie & Indivíduo & $\begin{array}{c}\text { Valores } \\
\text { fenotípicos }\end{array}$ & $\begin{array}{c}\text { Valores genéticos } \\
\text { (média }+ \text { efeito genético } \\
\text { aditivo predito) }\end{array}$ & $\begin{array}{c}\text { Ganho } \\
\text { genético }\end{array}$ & $\begin{array}{l}\text { Nova Média } \\
\text { da população } \\
\text { melhorada }\end{array}$ \\
\hline & & & & & $\mathrm{cm}$ & \\
\hline 1 & 8 & 2 & 163,00 & 109,42 & 15,53 & 109,42 \\
\hline 3 & 21 & 1 & 180,00 & 108,93 & 15,28 & 109,17 \\
\hline 3 & 2 & 6 & 179,00 & 108,34 & 15,01 & 108,90 \\
\hline 3 & 3 & 1 & 159,00 & 108,12 & 14,81 & 108,70 \\
\hline 1 & 8 & 2 & 151,00 & 106,72 & 14,42 & 108,31 \\
\hline 3 & 3 & 1 & 141,00 & 106,58 & 14,13 & 108,02 \\
\hline 1 & 19 & 2 & 152,00 & 106,56 & 13,92 & 107,81 \\
\hline 3 & 5 & 4 & 156,00 & 106,25 & 13,72 & 107,61 \\
\hline 1 & 19 & 2 & 139,00 & 106,04 & 13,44 & 107,33 \\
\hline 3 & 8 & 5 & 135,00 & 104,76 & 13,18 & 107,07 \\
\hline Média geral $(\mathrm{cm})$ & & & & & & 93,89 \\
\hline Acurácia seletiva & & & & & & 0,58 \\
\hline
\end{tabular}


Tabela 3. Valores fenotípicos, genéticos aditivos, ganhos genéticos preditos e nova média da população melhorada, das 10 melhores progênies para o caráter diâmetro ( $\mathrm{mm}$ ) em seringueira, no Município de Dois Irmãos do Buriti (MS)

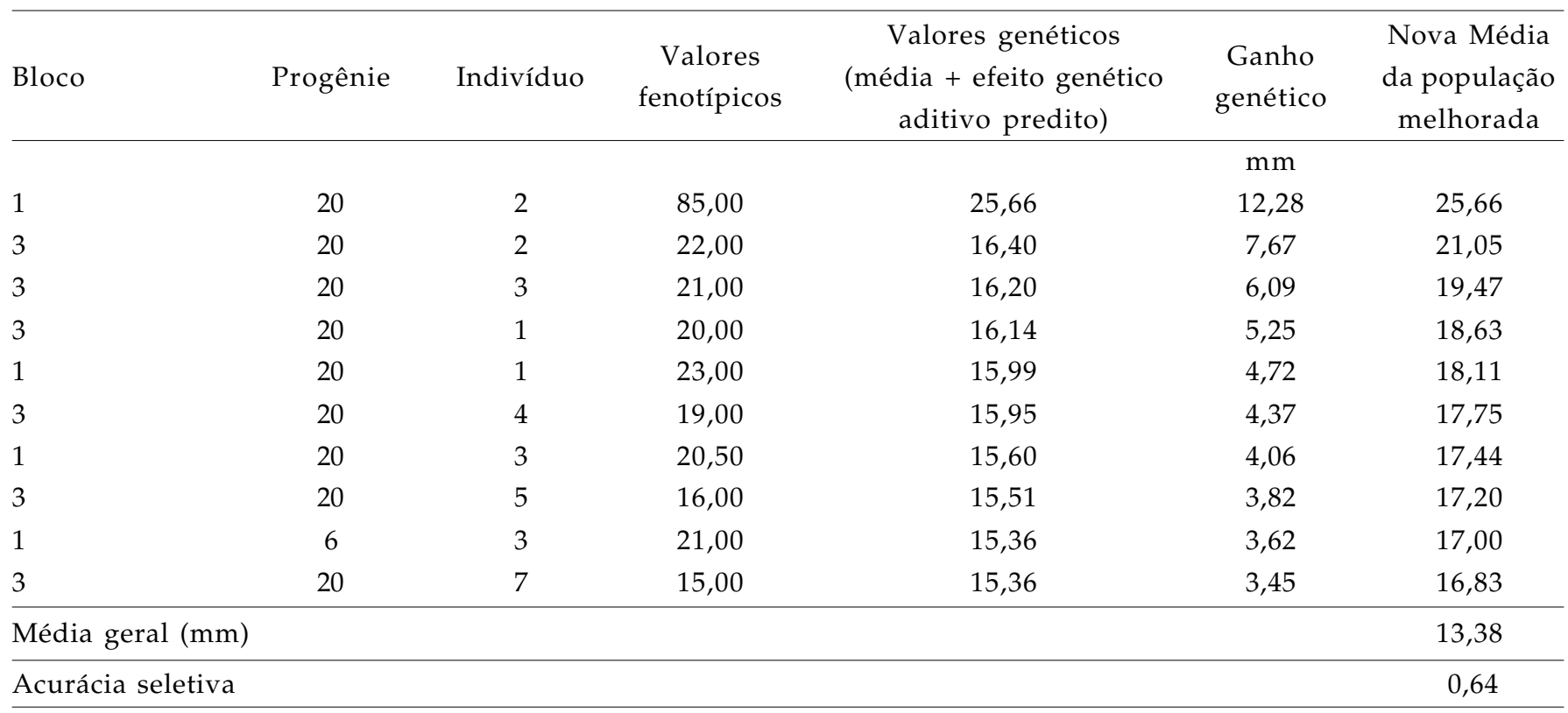

Tabela 4. Efeitos aditivos ( $\hat{a})$, valores genéticos aditivos preditos $(\hat{\mu}+\hat{a})$, efeitos genotípicos $(\hat{g})$ e valores genotípicos $(\hat{\mu}+\hat{g})$ preditos dos 10 melhores genótipos da seringueira aos 12 meses de idade para o caráter altura no município de Dois Irmãos do Buriti (MS)

\begin{tabular}{|c|c|c|c|c|c|c|c|c|c|}
\hline \multicolumn{4}{|c|}{ Propagação sexuada } & \multirow[b]{2}{*}{$\hat{\mu}+\hat{a}$} & \multicolumn{5}{|c|}{ Propagação assexuada } \\
\hline Bloco & Genótipo & Indivíduo & $\hat{\mathrm{a}}$ & & Bloco & Genótipo & Indivíduo & $\hat{\mathrm{g}}$ & $\hat{\mu}+\hat{g}$ \\
\hline 1 & 8 & 2 & 15,54 & 109,42 & 3 & 2 & 6 & 22,94 & 116,83 \\
\hline 3 & 21 & 1 & 15,04 & 108,93 & 3 & 21 & 1 & 22,80 & 116,69 \\
\hline 3 & 2 & 6 & 14,46 & 108,34 & 1 & 8 & 2 & 22,55 & 116,44 \\
\hline 3 & 3 & 1 & 14,24 & 108,12 & 2 & 5 & 4 & 20,25 & 114,14 \\
\hline 2 & 8 & 2 & 12,84 & 106,72 & 3 & 3 & 1 & 19,98 & 113,87 \\
\hline 1 & 3 & 1 & 12,69 & 106,58 & 2 & 19 & 2 & 18,23 & 112,12 \\
\hline 2 & 19 & 2 & 12,67 & 106,56 & 2 & 8 & 2 & 18,05 & 111,94 \\
\hline 2 & 5 & 4 & 12,36 & 106,25 & 1 & 3 & 1 & 17,40 & 111,29 \\
\hline 1 & 19 & 2 & 11,15 & 105,04 & 1 & 19 & 2 & 15,69 & 109,58 \\
\hline 3 & 8 & 5 & 10,87 & 104,76 & 3 & 8 & 5 & 14,78 & 108,67 \\
\hline Média & $1(\mathrm{~cm})$ & & & & & & & & 93,89 \\
\hline
\end{tabular}

Os valores genotípicos $(\hat{\mu}+\hat{g})$ preditos foram superiores aos genéticos aditivos $(\hat{\mu}+\hat{a})$, o que pode indicar maiores possibilidades de ganhos com a implantação de plantios clonais.

Os valores genéticos aditivos preditos $(\hat{\mu}+\hat{a})$ e genotípicos $(\hat{\mu}+\hat{g})$, também assumindo grau médio de dominância igual a 1 em uma população com nível intermediário de melhoramento, dos 10 melhores genótipos da seringueira aos 12 meses de idade para o caráter diâmetro, estão contidos na tabela 5 .

Observa-se que para o caráter diâmetro o material genético é comum (genótipos 20 e 6) e na mesma seqüência para propagação sexuada $(\hat{\mu}+\hat{a})$ e assexuada $(\hat{\mu}+\hat{g})$. 
Tabela 5. Efeitos aditivos (â), valores genéticos aditivos preditos $(\hat{\mu}+\hat{a})$, efeitos genotípicos $(\hat{g})$ e valores genotípicos $(\hat{\mu}+\hat{g})$ preditos dos 10 melhores genótipos da seringueira aos 12 meses de idade para o caráter diâmetro no município de Dois Irmãos do Buriti (MS)

\begin{tabular}{|c|c|c|c|c|c|c|c|c|c|}
\hline \multicolumn{4}{|c|}{ Propagação sexuada } & \multirow[b]{2}{*}{$\hat{\mu}+\hat{a}$} & \multicolumn{5}{|c|}{ Propagação assexuada } \\
\hline Bloco & Genótipo & Indivíduo & $\hat{\mathrm{a}}$ & & Bloco & Genótipo & Indivíduo & $\hat{\mathrm{g}}$ & $\hat{\mu}+\hat{g}$ \\
\hline 3 & 20 & 2 & 3,07 & 16,45 & 3 & 20 & 2 & 3,59 & 16,97 \\
\hline 3 & 20 & 3 & 2,91 & 16,30 & 3 & 20 & 3 & 3,33 & 16,71 \\
\hline 3 & 20 & 1 & 2,76 & 16,14 & 3 & 20 & 1 & 3,07 & 16,45 \\
\hline 1 & 20 & 1 & 2,61 & 16,00 & 1 & 20 & 1 & 2,82 & 16,20 \\
\hline 3 & 20 & 4 & 2,60 & 15,98 & 3 & 20 & 4 & 2,81 & 16,19 \\
\hline 1 & 20 & 3 & 2,22 & 15,60 & 1 & 6 & 3 & 2,73 & 16,11 \\
\hline 3 & 20 & 5 & 2,13 & 15,51 & 1 & 20 & 3 & 2,17 & 15,55 \\
\hline 1 & 6 & 3 & 1,98 & 15,36 & 3 & 20 & 5 & 2,03 & 15,41 \\
\hline 3 & 20 & 7 & 1,98 & 15,36 & 3 & 20 & 7 & 1,99 & 15,37 \\
\hline 2 & 20 & 3 & 1,93 & 15,31 & 2 & 20 & 3 & 1,95 & 15,33 \\
\hline Média & $(\mathrm{mm})$ & & & & & & & & 13,38 \\
\hline
\end{tabular}

De forma similar ao caráter altura, os valores genotípicos foram superiores aos genéticos aditivos, resultando possibilidades de ganhos com a implantação de pomares de plantios clonais.

Cabe ressaltar que a seleção dos melhores indivíduos, com base no sistema de propagação (assexuada ou sexuada), está intimamente ligada aos objetivos do programa de melhoramento da espécie. Caso o objetivo seja a transformação do teste de progênie em pomar de semente por mudas, os indivíduos devem ser selecionados com base em $(\hat{\mu}+\hat{a})$; caso o objetivo seja o fornecimento de material para instalação de testes clonais e, posteriormente, para estabelecimento de plantios clonais, então se deve selecionar os indivíduos com base em $(\hat{\mu}+\hat{g})$, para que ocorra a maximização do ganho genético.

\section{CONCLUSÕES}

1. A variabilidade genética demonstrada e as herdabilidades individuais e de progênies obtidas, estimulam a continuidade do programa de melhoramento genético, com a possibilidade de maximização dos ganhos nas gerações seguintes.

2. A seleção com base nos valores genéticos preditos, usando-se propagação sexuada, propicia maior progresso genético para os caracteres de crescimento.

\section{AGRADECIMENTOS}

Os autores agradecem à Prefeitura do Município de Dois Irmãos do Buriti (MS), pelo apoio logístico na instalação do experimento e às Instituições CNPq e CAPES pelas bolsas concedidas.

\section{REFERÊNCIAS}

ALIKA, J.E. Heritability and genotypic gain from selection rubber (Hevea brasiliensis). Silvae Genetica, Frankfurt, v.34, n. 1, p. 1-4, 1985.

BOOCK, M.V.; GONÇALVES, P.S.; BORTOLETTO, N.; MARTINS, A.L.M. Herdabilidade, variabilidade genética e ganhos genéticos para produção e caracteres morfológicos em progênies jovens de seringueira. Pesquisa Agropecuária Brasileira, Brasília, v.30, n.5, p.673-681, 1995.

COSTA, R.B.; RESENDE, M.D.V.; ARAUJO, A.J.;GONÇALVES, P. S.; BOTOLETTO, N. Seleção combinada univariada e multivariada aplicada ao melhoramento genético da seringueira. Pesquisa Agropecuária Brasileira, v.35, n.2, p. 381388, 2000a.

COSTA, R.B.; RESENDE, M.D.V.; GONÇALVES, P.S. Selection and genetic gain in populations of Hevea brasiliensis with a mixed mating system. Genetics and Molecular Biology, v.23,.3, p. $671-679,2000 \mathrm{~b}$.

COSTA, R.B.; RESENDE, M.D.V.; GONÇALVES, P.S.; ARRUDA, E. J.; OLIVEIRA, L. C. S.; BORTOLETTO, N. Prediction of genotypic values for yield in rubber tree-clone test trials using REML/BLUP procedure. Crop Breeding and Applied Biotechnology, Viçosa, v.2, n.4, p.575-582, 2002.

COSTA, R.B.; RESENDE, M.D.V.; CONTINI, A.Z.; REGO, F.L.H.; ROA, R.A.R.; MARTINS, W. J. Avaliação genética dentro de progênies de erva-mate (Ilex paraguariensis St. Hil.), na região de Caarapó, MS, pelo procedimento REML/BLUP. Ciência Florestal, Santa Maria, v.15, n.4, p.371-376, 2005. 
DASLIN, A.; BAIHAKI, A.; DANAKUSUMA, T.M.; HAERUMAN, M.S. Genotypes $x$ environment interaction in rubber and their implications in clonal selection. Bulletin Perkaretan, Bogor, n.4, p.23-28, 1986.

FALCONER, D. S. Introdução à genética quantitativa. Viçosa: Universidade Federal de Viçosa, 1987.

FURLANI, R.C.M.; MORAES, M.L.T.; RESENDE, M.D.V.; FURLANI JUNIOR, E.; GONÇALVES, P.S.; VALÉRIO FILHO, W.V.; PAIVA, J.R. Estimation of variance components and prediction of breeding values in rubber tree breeding usig the REML/BLUP procedure. Genetics and Molecular Biology, Ribeirão Preto, v.28, n.2, p.271-276, 2005.

GARCIA, C.H. Tabela para classificação do coeficiente de variação. Piracicaba: Instituto de Pesquisas e Estudos Florestais-IPEF, 1989. (Circular Técnica 171)

GILBERT, N.E.; DODDS, K.S.; SUBRAMANIAN, S. Progress of breeding investigations with Hevea brasiliensis. Analysis of data from earlier crosses. Journal of the Rubber Research Institute of Malaysia, Kuala Lumpur, v.3, n.5, p.365-380, 1973.

GONÇALVES, P.S.; BATAGLIA, O.C.; SANTOS, W.R.; ORTOLANI, A.A.; SEGNINI JUNIOR, I.; SHIKASHO, E.H. Growth trends, genotype-environment interaction and genetic gains in six-years old rubber tree clones (Hevea) in São Paulo State, Brazil. Genetics and Molecular Biology, n.21, p.115-122, 1998.

GONÇALVES, P.S.; MORAES, M.L.T.; BORTOLETTO, N.; COSTA, R.B.; GONÇALVES, E. C. P. Genetic variation in growth traits and yield rubber trees (Hevea brasiliensis) growing in the Brazilian State of São Paulo. Genetics and Molecular Biology, Ribeirão Preto, v. 28, n. 4, p. 765-772, 2005.

JAYASEKERA, N.E.M. A basis for selecting hevea clones stable to unpredictable agroclimatic variability. Silvae Genetica, Frankfort, n.32, p.181-185, 1983.

MEENATTOR, R.J.; VINOD K.K.; KRUSHNAKUMAR, A.K.; SETURAJ, M.R.; POTTY, S.N.; SINHÁ, R.R. Clone $x$ environment interaction during early growth phase of Hevea brasiliensis. i. clonal stability on diameter. Indian Journal of Natural Rubber Research, n.4, p.51-58, 1991.

MISSIO, R. F.; SILVA, A. M.; DIAS, L. A. S.; MORAES, M. L. T.; RESENDE, M. D. V. Estimates of genetic parameters and prediction of additive genetic values in Pinus kesya progenies. Crop Breeding and Applied Biotechnology, Viçosa, v.5, n.4, p. 394-401, 2005.

MORETI, D.; GONÇALVES, P. de S.; GORGULHO, E.P.; MARTINS, A.L.M.; BORTOLETTO, N. Estimativas de parâmetros genéticos e ganhos esperados com a seleção de caracteres juvenis em progênies de seringueira. Pesquisa Agropecuária Brasileira, Brasília, v.29, n.7, p.1099-1109, 1994.

NGA, B.H.; SUBRAMANIAN, S. Variation in Hevea brasiliensis. I. Yield and girth data of the 1937 hand pollinated seedlings. Journal of the Rubber Research Institute of Malaysia, Kuala Lumpur, v. 24, n.2, p.69-74, 1974.
OKNOPISE, O.U.; OLAPADA, O.; MEKAKO, H.U. Genotype X environment interaction in Hevea brasiliensis. Indian Journal of Genetics, n.46, p.506-511, 1986.

PAIVA, J.R.; MIRANDA FILHO, J.B.; SIQUEIRA, E.R.; VALOIS, A.C.C. Predição do ganho de alguns caracteres em seringueira em três esquemas de seleção. Pesquisa Agropecuária Brasileira, Brasília, v.17, n.11, p.1646-1653, 1982.

PEREIRA, J.P. Seringueira: formação de mudas, manejo e perspectivas no noroeste do Paraná. Londrina: IAPAR, 1992. 60p. (IAPAR. Circular Técnica, 700).

RESENDE, M.D.V. Genética biométrica e estatística no melhoramento de plantas perenes. Brasília: Embrapa Informação Tecnológica. 975p. 2002a.

RESENDE, M.D.V. Software SELEGEN - REML/BLUP. Colombo: Embrapa Florestas, 2002b. 67p. (Embrapa Florestas - Documentos, 77)

REZENDE, M.D.V.; DIAS, L.A.S. Aplicação da metodologia de modelos mistos (REML/BLUP) na estimação de parâmetros genéticos e predição de valores genéticos e aditivos e genotípicos em espécies frutíferas. Revista Brasileira de Fruticultura, v.22, n.1, p.44-52, 2000.

RESENDE, M.D.V.; FERNANDES, J.S.C. Procedimento BLUP (Melhor Predição Linear Não Viciada) individual para delineamentos experimentais aplicados ao melhoramento florestal. Revista Matemática e Estatística, São Paulo, n.17, p.89-107, 1999.

SIMEÃO, R.M.; STURION, J.A.; RESENDE, M.D.V. Avaliação Genética em erva-mate pelo procedimento BLUP individual multivariado sob interação genótipo $x$ ambiente. Pesquisa Agropecuária Brasileira, Brasília, v. 37, n.11, p.1589-1596, 2002.

TAN, H. Genotype x environment interaction studies In rubber (Hevea) clones. Journal of the Rubber Research Institute of Malaysia,. Kuala Lumpur, n. 10, p. 63-76, 1995.

VENCOVSKY, R.; BARRIGA, P. Genética biométrica no fitomelhoramento. Ribeirão Preto: Sociedade Brasileira de Genética, 1992. 496p. 